\title{
Research on Definition and Risk Assessment of Near Miss Events in Coal Mine
}

\author{
Sun Shuying \\ College of Energy Science and Engineering \\ Xi'an University of Science and Technology \\ Xi'an,China \\ e-mail: 1621435456@qq.com
}

\begin{abstract}
At present, extraordinarily serious casualties in coal mine is often pay more attention, however, a large number of minor accidents, especially these events caused no casualties or economic loss is not seriously enough attention. Which lead to neglect or even let near miss and the presence of attempted events. In this study, the near miss of mines was definite, and its difference and relationship with hazards and risks was analyzed. Drawing on traditional LEC risk assessment methods, relative risk assessment model of near miss was constructed by using $f$ risk index, practice has proved that the classification results are basically the same with the traditional LEC risk assessment methods, and it can reflect the risk characteristics of near miss events from the many perspectives. Which has great practical significance in eliminating all kinds of production safety hazards in coal mine, preventing the occurrence of major accidents in practical work.
\end{abstract}

Keywords- near miss; hazard; risk assessment; $f$ index; coal mine

\section{INTRODUCTION}

The safety issues of coal mine production in China has drawn attention from all walks of life for a long time. In recent years, the government and coal companies have done a lot of work in the prevention and treatment of mine casualties, production safety accidents and fatalities of coal mine declined overall. But extraordinarily serious casualties in coal mine is often pay more attention, however, a large number of minor accidents, especially these events caused no casualties or economic loss is not seriously enough attention. Which lead to neglect or even let near miss and the presence of attempted events. Measures that should be taken did not to take, or taken measures were not sufficient to eliminate the risk, which make the dangerous situation be in a continuous state situation, and many accidents that should be avoided did not be avoided. If monitoring and diagnostics for the risk factors can be previously strengthen, warning message is issued before the sign of an accident but not yet formed the accident, corresponding measures was immediately taken to eliminate the risk, then it will play a proactive effect.

The aim of his study was to analysis and define the near miss, then the near miss of coal mine (especially near miss caused by human factors) were graded taking an improved LEC risk assessment method, In order to explore measures to prevent accidents, and to avoid the occurrence of major coal mine accidents in the maximum.

\section{DEFINITION OF NEAR MISS EVENTS}

\section{A. The understanding of concept for near miss}

In 1930s, Heinrich pointed out the ratio of serious injury, minor injury and non-injury accident was 1:29:300 by statistics a large number of accident. Bode also found that the ratio of serious injury, minor injury, property damage and no loss and injury accident was 1:10:30:600 in the survey. Relative to the accident that has a very serious consequences, the impact of a large number of incidents was quite limited, even the event did not cause more injury or property damage. Within a certain period of time or a certain range, with diminishing the severity of the consequences, the number of accident is increasing, it showed a pyramid-type distribution. Near miss is the accident which did not cause a lot of damage or loss at the bottom of the pyramid.

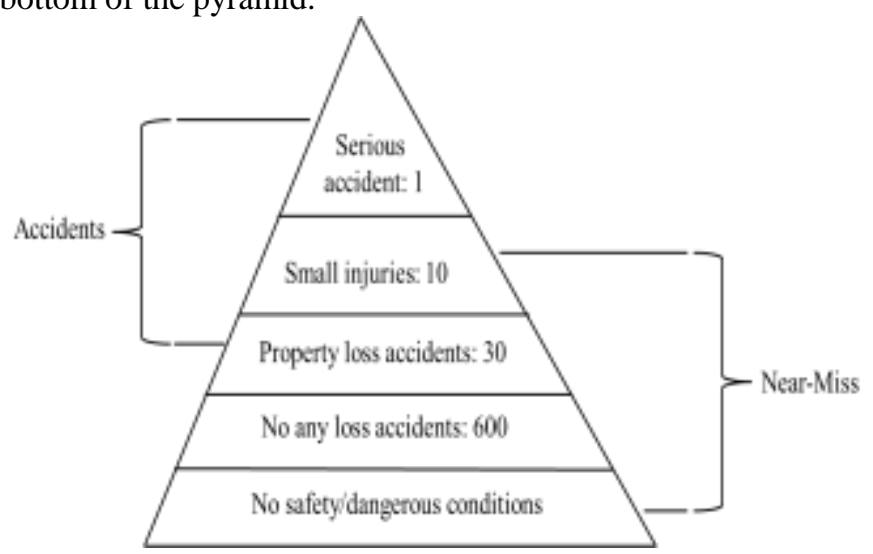

Figure 1. Security pyramid model

Early the definition of near miss tends to accident theory, it was called the near miss accident. But the accident has a loss, near miss is little incident with no loss or a slight loss. Therefore, the near miss incident is more scientific over the near miss accident ${ }^{[1]}$.

On the basis of previous academic studies, the concept of near miss was clearly defined in this study. The near miss of coal mine referred to small events that has a 
characteristic symptom of accidents, but did not produce losses and injuries or only a slight loss and damage due to lack of conditions. If the conditions changed, there will still be the possibility resulting in an accident

\section{B. The difference and relationship between Near-Miss, hazard and risk}

Hazards and accidents have in common, they are likely to cause the accident, but they are not equivalent concepts. Tian Shuicheng ${ }^{[2]}$ raised the view of three types hazards in 2001. The first type hazard was energy carriers or energy source; the second type hazard was the fault substance, physical environmental factors and individual human behavior mistakes; the third type hazard was organizational factors that are not consistent with the safety, including not safe behavior and mistakes, etc. And identification and control for the third type hazard were studied, which further deepen the understanding of hazards. Hazard reflected the objectivity of danger, some of the first type hazard must exist due to the need to produce. But the accidents did not necessarily exist.

Near miss is the accident between the cause of the accident and injury events, and it did not develop into injury events. But no matter it is an accident or near miss, it has an objective existence of hazardous substances or energy carriers (hazard belongs to the first category), which is a prerequisite for occurrence of near miss or accident. And there exists the second type hazard, such as people, objects and environments, it may lead to the release of hazardous substances or energy, this is the direct cause of near miss or the incident. The third type hazards or management deficiencies and organizational failures of potential hazards can be seen as an indirect cause of near miss or the accidents. The relationships between near miss and hazard and risk are shown in Figure 2.

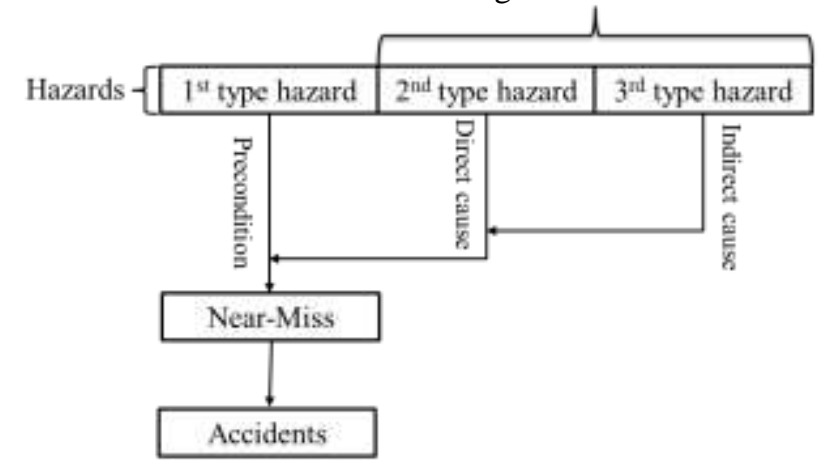

Figure 2. The relationship between near-miss, hazard and risk

\section{RISK ASSESSMENT OF NEAR-MISS}

In 2010, Shi Xiaohong divided attempted events into three categories based on the main cause of the event: attempted events caused by unsafe behavior, such as misuse or violation of rules; attempted event caused by unsafe condition, such as corrosion and aging; and attempted event caused by insecurity environmental and management deficiencies. According to the risk degree and consequences of attempted events, it can be divided into two levels: general attempted events and larger attempted events. General attempted event is an event that could lead to an accident of the enterprise; larger attempted event is an event whose potential consequences is likely to result in group stage accident ${ }^{[3]}$.

In 2003, Phimister ${ }^{[4,5]}$ attempted to divided attempted events into seven categories: people's unsafe behavior; unsafe condition of matter; gradually serious tiny events; causing harm events in the future (did not happen now); resulting in property damage incidents; impact protection barrier events; potentially damage the environment events. In 2002, Ritwlk ${ }^{[6]}$ proposed a risk-based management approach based on attempted event, the method will attempt to divide attempted events into unsafe state of matter and people's unsafe behavior.

Although the above methods can be used to qualitatively classify near miss events, but this method is difficult to achieve accurate qualitative classification during the actual operation, it is not conducive to management of near miss events and the prevention of accidents. Based on LEC evaluation method, combined with the actual situation of coal enterprises, the risk of near miss was assessed by using five areas indicators related to the risk rate index of the system. These five indicators are: the possibility of near miss events leading to an accident, L; the frequent of personnel exposure to hazardous environments, E; the monitor extent of the event, $\mathrm{M}$; the learn value of events, $S$; probable consequences of an accident once occurred, C. According to the characteristics of the five risk indicators, combined with LEC risk assessment, each risk indicator will be divided into seven levels for statistical analysis, specific criteria for the classification was shown in Table 1 .

TABLE I. GRADING STANDARD OF RISK INDICATORS

\begin{tabular}{|c|c|c|c|c|c|c|c|}
\hline \multirow{2}{*}{$\begin{array}{c}\begin{array}{c}\text { Risk } \\
\text { indicators }\end{array} \\
\text { L., }\end{array}$} & \multicolumn{7}{|c|}{ Grading standard (score). } \\
\hline & $\begin{array}{l}\text { Completely } \\
\text { possible }(10)\end{array}$ & $\begin{array}{l}\text { Quite } \\
\text { possible(6). }\end{array}$ & $\begin{array}{c}\text { Possible, but } \\
\text { not often } \\
(3) .\end{array}$ & 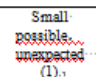 & Impossible(0.5). & $\begin{array}{c}\begin{array}{c}\text { Highly } \\
\text { impossible } \\
(0.2) .\end{array} \\
\text {. }\end{array}$ & $\begin{array}{c}\text { Actually: } \\
\text { impossible } \\
(0.1):=\end{array}$ \\
\hline E. & $\begin{array}{l}\text { Continued } \\
\text { exposure (10). }\end{array}$ & $\begin{array}{l}\text { Wodking } \\
\text { time } \\
\text { exposure } \\
(\sigma)\end{array}$ & $\begin{array}{r}\text { Exposure once } \\
\text { a weel: - (3)." }\end{array}$ & $\begin{array}{l}\text { Exposure } \\
\text { Once a month } \\
\text { (1). }\end{array}$ & $\begin{array}{c}\text { Exposure } \\
\text { several times a } \\
\text { year }(0.5) .\end{array}$ & $\begin{array}{c}\text { rare } \\
\text { expousure } \\
(0.2) .\end{array}$ & $\begin{array}{c}\text { No } \\
\text { expossure. } \\
(0.1) .\end{array}$ \\
\hline M. & $\begin{array}{l}\text { completely } \\
\text { monitor (10). }\end{array}$ & $\begin{array}{c}\text { Most } \\
\text { monitor(()). }\end{array}$ & $\begin{array}{c}\text { Occasionally } \\
\text { monitor (3). }\end{array}$ & $\begin{array}{l}\text { mot easy to } \\
\text { monitor(3). }\end{array}$ & $\begin{array}{l}\text { Impossible } \\
\text { monitor }(0.5) .\end{array}$ & $\begin{array}{c}\text { Highly } \\
\text { impossible } \\
\text { monitor } \\
(0.2) .\end{array}$ & $\begin{array}{c}\text { Actually } \\
\text { impossible } \\
\text { monitor } \\
(0.1) .\end{array}$ \\
\hline s.r. & $\begin{array}{l}\text { greast Laam } \\
\text { value (10). }\end{array}$ & $\begin{array}{l}\text { Large leam. } \\
\text { value(6). }\end{array}$ & $\begin{array}{c}\text { Certain laam } \\
\text { value(3) }\end{array}$ & $\begin{array}{l}\text { Less leam } \\
\text { value(3). }\end{array}$ & $\begin{array}{c}\text { Small leam } \\
\text { value( }(0.5) .\end{array}$ & $\begin{array}{l}\begin{array}{l}\text { No leam } \\
\text { value(0.2). }\end{array} \\
\end{array}$ & $\begin{array}{l}\text { Actually } \\
\text { no leam } \\
\text { valua( } 0.1) \text {. }\end{array}$ \\
\hline c. & $\begin{array}{c}\text { 10. people } \\
\text { desd.: } \\
(10) .\end{array}$ & $\begin{array}{c}\text { 3\%9. prople } \\
\text { dead(6).. }\end{array}$ & $\begin{array}{c}1 \sim 2 \text { paople } \\
\text { deadi. } \\
\text { (3).: }\end{array}$ & $\begin{array}{l}\text { Serious.1 } \\
\text { (3).. }\end{array}$ & $\begin{array}{c}\text { Significant } \\
\text { dissbility(0.5).n }\end{array}$ & $\begin{array}{c}\text { Attractive } \\
(0.2) .\end{array}$ & $\begin{array}{c}\text { no } \\
\text { consequen } \\
\text { ces(0.1).. }\end{array}$ \\
\hline
\end{tabular}

In order to better reflect that the risk of near miss incidents is relative, this study $f$ index was used to represent the relative risk of near miss incidents, namely:

$$
\begin{gathered}
f_{i}=\sum_{j=1}^{7}\left(\left|100 \times \frac{h_{i j}-q_{i j}}{q_{i j}}\right|-\right. \\
\left.\frac{1}{n} \sum_{i=1}^{n}\left|100 \times \frac{h_{i j}-q_{i j}}{q_{i j}}\right|\right) .
\end{gathered}
$$

Where, $f$ was the dimensionless; $h_{i j}$ was the overall score of $j$-th indicator for the $i$-th near miss event; $q_{i j}$ was the lowest value of the $j$-th indicators in the $i$-th near miss event; $n$ is the number of near miss incidents, herein, $n=30$. $F$ values were shown in Table 2 . 
TABLE II THE $F$ VALUE

\begin{tabular}{|c|c|c|c|c|c|c|c|}
\hline $\begin{array}{l}\mathbf{N} \\
\mathbf{0}\end{array}$ & Near-Miss & $\mathbf{L}$ & $\mathbf{E}$ & $\mathbf{M}$ & $\mathbf{S}$ & $\mathbf{C}$ & $f i$ \\
\hline 1 & $\begin{array}{l}\text { Replace cutter } \\
\text { tooth of roller } \\
\text { when the coal } \\
\text { stoker was } \\
\text { stopped and } \\
\text { locked. }\end{array}$ & $\begin{array}{l}2 \\
8 \\
9 . \\
5 \\
2\end{array}$ & $\begin{array}{c}39 \\
4 . \\
72\end{array}$ & $\begin{array}{l}8 \\
3 \\
1 . \\
0 \\
3\end{array}$ & $\begin{array}{l}4 \\
1 \\
6 . \\
7 \\
6\end{array}$ & $\begin{array}{c}33 \\
0 . \\
34\end{array}$ & $\begin{array}{c}- \\
14.6 \\
0\end{array}$ \\
\hline 2 & $\begin{array}{l}\text { Charged } \\
\text { maintenance, } \\
\text { relocation } \\
\text { equipment }\end{array}$ & $\begin{array}{l}5 \\
9 \\
0 . \\
7 \\
5\end{array}$ & $\begin{array}{r}19 \\
92 \\
.3 \\
5\end{array}$ & $\begin{array}{l}8 \\
1 \\
4 . \\
3 \\
9\end{array}$ & $\begin{array}{l}4 \\
2 \\
8 . \\
1 \\
5\end{array}$ & $\begin{array}{l}10 \\
79 \\
.7 \\
8\end{array}$ & $\begin{array}{c}18.4 \\
4\end{array}$ \\
\hline 3 & $\begin{array}{l}\text { Staff taking a } \\
\text { rest on the } \\
\text { arm when } \\
\text { shearer } \\
\text { shutdown, or } \\
\text { sleep leaning } \\
\text { on the arm }\end{array}$ & $\begin{array}{l}4 \\
2 \\
6 . \\
8 \\
7\end{array}$ & $\begin{array}{l}11 \\
41 \\
.6 \\
9\end{array}$ & $\begin{array}{l}8 \\
3 \\
2 . \\
2 \\
2\end{array}$ & $\begin{array}{l}3 \\
7 \\
9 . \\
4 \\
2\end{array}$ & $\begin{array}{c}27 \\
9 . \\
78\end{array}$ & 4.63 \\
\hline 4 & $\begin{array}{c}\text { Stoker } \\
\text { Walking, } \\
\text { dragging and } \\
\text { hanging } \\
\text { cables }\end{array}$ & $\begin{array}{l}5 \\
9 \\
4 . \\
2 \\
7\end{array}$ & $\begin{array}{r}19 \\
92 \\
.3 \\
5\end{array}$ & $\begin{array}{l}7 \\
5 \\
9 . \\
6 \\
9\end{array}$ & $\begin{array}{l}2 \\
7 \\
0 . \\
6 \\
4\end{array}$ & $\begin{array}{c}52 \\
4 . \\
72\end{array}$ & 8.89 \\
\hline 5 & $\begin{array}{l}\text { No stop and } \\
\text { latch bolt } \\
\text { machine, } \\
\text { person drill } \\
\text { pipe or duct } \\
\text { between the } \\
\text { two treatment } \\
\text { arms }\end{array}$ & $\begin{array}{l}5 \\
5 \\
9 . \\
0 \\
3\end{array}$ & $\begin{array}{c}16 \\
96 \\
.8 \\
3\end{array}$ & $\begin{array}{l}8 \\
3 \\
2 . \\
2 \\
2\end{array}$ & $\begin{array}{l}4 \\
3 \\
1 . \\
5 \\
0\end{array}$ & $\begin{array}{l}81 \\
2 . \\
36\end{array}$ & $\begin{array}{c}11.2 \\
7\end{array}$ \\
\hline 6 & $\begin{array}{l}\text { Do not close } \\
\text { the air door, } \\
\text { while open } \\
\text { two doors, } \\
\text { causing short } \\
\text { circuit of } \\
\text { Merry }\end{array}$ & $\begin{array}{l}5 \\
6 \\
6 . \\
0 \\
8\end{array}$ & $\begin{array}{c}13 \\
70 \\
.7 \\
1\end{array}$ & $\begin{array}{l}8 \\
4 \\
1 . \\
7 \\
4\end{array}$ & $\begin{array}{l}4 \\
3 \\
0 . \\
8 \\
3\end{array}$ & $\begin{array}{c}68 \\
4 . \\
27\end{array}$ & 5.79 \\
\hline 7 & $\begin{array}{l}\text { Free to get rid } \\
\text { of power } \\
\text { lockout of gas } \\
\text { and wind } \\
\text { power lockout } \\
\text { device }\end{array}$ & $\begin{array}{l}2 \\
6 \\
9 . \\
1 \\
6\end{array}$ & $\begin{array}{c}23 \\
7 . \\
20\end{array}$ & $\begin{array}{l}1 \\
9 \\
5 . \\
4 \\
8\end{array}$ & $\begin{array}{l}6 \\
1 . \\
3 \\
9\end{array}$ & $\begin{array}{c}48 \\
6 . \\
97\end{array}$ & $\begin{array}{c}- \\
27.2 \\
5\end{array}$ \\
\hline 8 & $\begin{array}{l}\text { Monitoring } \\
\text { equipment } \\
\text { failure for a } \\
\text { long time } \\
\text { without } \\
\text { treatment and } \\
\text { reporting }\end{array}$ & $\begin{array}{l}8 \\
2 . \\
4 \\
7\end{array}$ & $\begin{array}{r}17 \\
52 \\
.2 \\
4\end{array}$ & $\begin{array}{l}7 \\
7 \\
6 . \\
1 \\
0\end{array}$ & $\begin{array}{l}7 \\
1 . \\
9 \\
8\end{array}$ & $\begin{array}{c}86 \\
0 . \\
00\end{array}$ & 1.40 \\
\hline 9 & $\begin{array}{l}\text { Maintenance } \\
\text { of mining face } \\
\text { not cut the } \\
\text { power and } \\
\text { locked }\end{array}$ & $\begin{array}{l}5 \\
2 \\
4 . \\
6 \\
7\end{array}$ & $\begin{array}{l}10 \\
41 \\
.6 \\
9\end{array}$ & $\begin{array}{l}6 \\
9 \\
1 . \\
9 \\
1\end{array}$ & $\begin{array}{l}5 . \\
5 \\
6\end{array}$ & $\begin{array}{c}80 \\
5 . \\
62\end{array}$ & 4.51 \\
\hline $\begin{array}{l}1 \\
0\end{array}$ & $\begin{array}{c}\text { Riding } \\
\text { running belt }\end{array}$ & $\begin{array}{l}5 \\
5 \\
9 . \\
0 \\
3\end{array}$ & $\begin{array}{c}18 \\
70 \\
.9 \\
8\end{array}$ & $\begin{array}{l}7 \\
4 \\
2 . \\
5 \\
7\end{array}$ & $\begin{array}{l}2 \\
6 \\
9 . \\
1 \\
0\end{array}$ & $\begin{array}{c}90 \\
0 . \\
00\end{array}$ & $\begin{array}{c}11.3 \\
9\end{array}$ \\
\hline 1 & Not rinse coal & 6 & 21 & 7 & 0. & 24 & - \\
\hline
\end{tabular}

\begin{tabular}{|c|c|c|c|c|c|c|c|}
\hline 1 & $\begin{array}{c}\text { bunker clear } \\
\text { to overhaul } \\
\text { into the } \\
\text { bunker }\end{array}$ & $\begin{array}{l}4 . \\
9 \\
3\end{array}$ & $\begin{array}{l}7 . \\
15\end{array}$ & $\begin{array}{l}8 \\
2 . \\
4 \\
0\end{array}$ & $\begin{array}{l}0 \\
0\end{array}$ & $\begin{array}{l}7 . \\
64\end{array}$ & $\begin{array}{c}26.4 \\
8\end{array}$ \\
\hline $\begin{array}{l}1 \\
2\end{array}$ & $\begin{array}{c}\text { Start belt } \\
\text { falsely; not } \\
\text { alarm to start } \\
\text { belt }\end{array}$ & $\begin{array}{l}5 \\
4 \\
4 . \\
2 \\
3\end{array}$ & $\begin{array}{l}14 \\
35 \\
.6 \\
2\end{array}$ & $\begin{array}{l}7 \\
1 \\
8 . \\
5 \\
5\end{array}$ & $\begin{array}{l}3 \\
7 \\
0 . \\
9 \\
1\end{array}$ & $\begin{array}{c}34 \\
8 . \\
99\end{array}$ & 0.15 \\
\hline $\begin{array}{l}1 \\
3\end{array}$ & $\begin{array}{l}\text { Personnel } \\
\text { across while } \\
\text { belt and } \\
\text { crusher are } \\
\text { operating }\end{array}$ & $\begin{array}{l}5 \\
0 \\
0 . \\
5 \\
3 \\
\end{array}$ & $\begin{array}{c}74 \\
2 . \\
48\end{array}$ & $\begin{array}{l}5 \\
4 \\
7 . \\
0 \\
9\end{array}$ & $\begin{array}{l}3 \\
2 \\
8 . \\
4 \\
9\end{array}$ & $\begin{array}{c}29 \\
3 . \\
71\end{array}$ & $\begin{array}{c}- \\
12.7 \\
3\end{array}$ \\
\hline $\begin{array}{l}1 \\
4\end{array}$ & $\begin{array}{l}\text { After coal } \\
\text { excavation is } \\
\text { completed, } \\
\text { the driver } \\
\text { observed } \\
\text { tunneling } \\
\text { under the } \\
\text { empty top }\end{array}$ & $\begin{array}{l}4 \\
0 \\
5 . \\
2 \\
0\end{array}$ & $\begin{array}{c}31 \\
6 \\
6\end{array}$ & $\begin{array}{l}0 . \\
0 \\
0\end{array}$ & $\begin{array}{l}2 \\
7 \\
0 . \\
4 \\
4\end{array}$ & $\begin{array}{c}30 \\
2 . \\
70\end{array}$ & $\begin{array}{c}- \\
30.2 \\
6\end{array}$ \\
\hline $\begin{array}{l}1 \\
5\end{array}$ & $\begin{array}{l}\text { Imperfect } \\
\text { equipment } \\
\text { management, } \\
\text { such as not } \\
\text { timely } \\
\text { maintenance } \\
\text { or } \\
\text { replacement }\end{array}$ & $\begin{array}{l}5 \\
5 \\
4 . \\
6 \\
3\end{array}$ & $\begin{array}{c}18 \\
70 \\
.9 \\
8\end{array}$ & $\begin{array}{l}8 \\
4 \\
1 . \\
7 \\
4\end{array}$ & $\begin{array}{l}3 \\
4 \\
6 . \\
5 \\
8\end{array}$ & $\begin{array}{c}34 \\
0 . \\
90\end{array}$ & 6.55 \\
\hline $\begin{array}{l}1 \\
6\end{array}$ & $\begin{array}{l}\text { Using unsafe } \\
\text { equipment, } \\
\text { such as non- } \\
\text { explosion- } \\
\text { proof } \\
\text { electrical } \\
\text { equipment }\end{array}$ & $\begin{array}{c}5 \\
8 \\
0 . \\
1 \\
8\end{array}$ & $\begin{array}{c}19 \\
05 \\
.2 \\
8\end{array}$ & $\begin{array}{l}7 \\
8 \\
7 . \\
0 \\
4\end{array}$ & $\begin{array}{l}3 \\
0 \\
2 . \\
6 \\
1\end{array}$ & $\begin{array}{c}11 \\
35 \\
.9 \\
6\end{array}$ & $\begin{array}{c}16.0 \\
1\end{array}$ \\
\hline $\begin{array}{l}1 \\
7\end{array}$ & $\begin{array}{l}\text { Command } \\
\text { workers to } \\
\text { take risks, } \\
\text { such as } \\
\text { stopping the } \\
\text { wind not to } \\
\text { withdrawal of } \\
\text { workers } \\
\text { underground }\end{array}$ & $\begin{array}{l}5 \\
9 \\
4 . \\
2 \\
7\end{array}$ & $\begin{array}{c}19 \\
05 \\
.8 \\
0\end{array}$ & $\begin{array}{l}8 \\
4 \\
1 . \\
7 \\
4\end{array}$ & $\begin{array}{l}3 \\
9 \\
0 . \\
6 \\
8\end{array}$ & $\begin{array}{c}13 \\
58 \\
.4 \\
3\end{array}$ & $\begin{array}{c}20.7 \\
5\end{array}$ \\
\hline $\begin{array}{l}1 \\
8\end{array}$ & $\begin{array}{c}\text { Unreasonable } \\
\text { work } \\
\text { assignment, } \\
\text { coordinating } \\
\text { work not in } \\
\text { place } \\
\end{array}$ & $\begin{array}{l}0 . \\
0 \\
0\end{array}$ & $\begin{array}{c}0 . \\
00\end{array}$ & $\begin{array}{l}4 \\
2 \\
8 . \\
1 \\
8\end{array}$ & $\begin{array}{l}2 \\
4 \\
9 . \\
7 \\
3\end{array}$ & $\begin{array}{c}57 \\
0 . \\
11\end{array}$ & $\begin{array}{c}- \\
27.2 \\
8\end{array}$ \\
\hline $\begin{array}{l}1 \\
9\end{array}$ & $\begin{array}{l}\text { Cross-border } \\
\text { mining }\end{array}$ & $\begin{array}{l}5 \\
1 \\
3 . \\
3 \\
9\end{array}$ & $\begin{array}{r}14 \\
84 \\
.7 \\
0\end{array}$ & $\begin{array}{l}7 \\
9 \\
9 . \\
1 \\
7\end{array}$ & $\begin{array}{l}4 \\
1 \\
6 . \\
0 \\
9\end{array}$ & $\begin{array}{c}67 \\
0 . \\
79\end{array}$ & 5.67 \\
\hline $\begin{array}{l}2 \\
0\end{array}$ & $\begin{array}{c}\text { No } \\
\text { construction } \\
\text { program, } \\
\text { disorderly } \\
\text { exploitation } \\
\end{array}$ & $\begin{array}{l}5 \\
6 \\
7 . \\
8 \\
4\end{array}$ & $\begin{array}{c}14 \\
81 \\
.0 \\
0\end{array}$ & $\begin{array}{l}7 \\
7 \\
3 . \\
9 \\
6\end{array}$ & $\begin{array}{l}3 \\
9 \\
4 . \\
6 \\
4\end{array}$ & $\begin{array}{c}47 \\
3 . \\
03\end{array}$ & 3.25 \\
\hline $\begin{array}{l}2 \\
1\end{array}$ & $\begin{array}{l}\text { No clear } \\
\text { punishment } \\
\text { rules about } \\
\text { the safety }\end{array}$ & $\begin{array}{l}4 \\
3 \\
9 . \\
3\end{array}$ & $\begin{array}{c}18 \\
9 . \\
97\end{array}$ & $\begin{array}{l}7 \\
8 \\
1 . \\
0\end{array}$ & $\begin{array}{l}3 \\
5 \\
2 . \\
0\end{array}$ & $\begin{array}{c}0 . \\
00\end{array}$ & $\begin{array}{c}- \\
20.8 \\
5\end{array}$ \\
\hline
\end{tabular}




\begin{tabular}{|c|c|c|c|c|c|c|c|}
\hline & $\begin{array}{l}\text { aspects of the } \\
\text { accident }\end{array}$ & 0 & & 9 & 1 & & \\
\hline $\begin{array}{l}2 \\
2\end{array}$ & $\begin{array}{l}\text { No specific } \\
\text { regulations } \\
\text { about safety } \\
\text { rewards }\end{array}$ & $\begin{array}{l}3 \\
3 \\
6 . \\
9 \\
2 \\
\end{array}$ & $\begin{array}{c}11 \\
03 \\
.9 \\
6\end{array}$ & $\begin{array}{c}7 \\
1 \\
2 . \\
1 \\
3 \\
\end{array}$ & $\begin{array}{c}3 \\
6 \\
8 . \\
1 \\
0\end{array}$ & $\begin{array}{c}14 \\
0 . \\
90\end{array}$ & 9. \\
\hline $\begin{array}{l}2 \\
3\end{array}$ & $\begin{array}{l}\text { No regular } \\
\text { safety } \\
\text { education and } \\
\text { training }\end{array}$ & $\begin{array}{l}5 \\
7 \\
7 . \\
5 \\
3\end{array}$ & $\begin{array}{l}18 \\
89 \\
.4 \\
5\end{array}$ & $\begin{array}{l}7 \\
9 \\
8 . \\
9 \\
3\end{array}$ & $\begin{array}{l}3 \\
9 \\
1 . \\
3 \\
5\end{array}$ & $\begin{array}{c}75 \\
2 . \\
13\end{array}$ & $\begin{array}{c}12.2 \\
4\end{array}$ \\
\hline $\begin{array}{l}2 \\
4\end{array}$ & $\begin{array}{l}\text { Inadequate } \\
\text { hazard } \\
\text { symbol set in } \\
\text { workplace }\end{array}$ & $\begin{array}{l}5 \\
5 \\
0 . \\
2 \\
2\end{array}$ & $\begin{array}{l}19 \\
10 \\
.5 \\
5\end{array}$ & $\begin{array}{l}8 \\
2 \\
6 . \\
2 \\
8\end{array}$ & $\begin{array}{l}3 \\
9 \\
8 . \\
7 \\
3\end{array}$ & $\begin{array}{c}40 \\
8 . \\
31\end{array}$ & 8.29 \\
\hline $\begin{array}{l}2 \\
5\end{array}$ & $\begin{array}{l}\text { No lockout } \\
\text { and lock the } \\
\text { conveyor } \\
\text { when Coal } \\
\text { machine is } \\
\text { repairing, and } \\
\text { picks are } \\
\text { replacing, no } \\
\text { latch cradle } \\
\text { within scope } \\
\text { for the } \\
\text { maintenance }\end{array}$ & $\begin{array}{l}5 \\
3 \\
2 . \\
6 \\
0\end{array}$ & $\begin{array}{c}19 \\
63 \\
.3 \\
2\end{array}$ & $\begin{array}{l}8 \\
1 \\
0 . \\
8 \\
2\end{array}$ & $\begin{array}{l}3 \\
9 \\
6 . \\
7 \\
8\end{array}$ & $\begin{array}{c}64 \\
8 . \\
31\end{array}$ & $\begin{array}{c}11.5 \\
2\end{array}$ \\
\hline $\begin{array}{l}2 \\
6\end{array}$ & $\begin{array}{c}\text { Secretly } \\
\text { dumped } \\
\text { scraper, } \\
\text { loader lockout } \\
\text { protection or } \\
\text { shortened } \\
\text { lockout } \\
\text { protection } \\
\text { distance }\end{array}$ & $\begin{array}{l}4 \\
6 \\
9 . \\
1 \\
6\end{array}$ & $\begin{array}{c}10 \\
67 \\
.2 \\
8\end{array}$ & $\begin{array}{l}7 \\
8 \\
2 . \\
2 \\
8\end{array}$ & $\begin{array}{l}4 \\
1 \\
2 . \\
0 \\
6\end{array}$ & $\begin{array}{c}96 \\
5 . \\
17\end{array}$ & 3.32 \\
\hline $\begin{array}{l}2 \\
7\end{array}$ & $\begin{array}{l}\text { Transmission } \\
\text { power by } \\
\text { lines the fault } \\
\text { is not } \\
\text { excluded }\end{array}$ & $\begin{array}{l}5 \\
8 \\
8 . \\
1 \\
1\end{array}$ & $\begin{array}{l}19 \\
58 \\
.0 \\
5\end{array}$ & $\begin{array}{l}7 \\
8 \\
9 . \\
5 \\
4\end{array}$ & $\begin{array}{c}4 \\
2 \\
8 . \\
1 \\
5\end{array}$ & $\begin{array}{l}11 \\
26 \\
.9 \\
7\end{array}$ & $\begin{array}{c}18.2 \\
5\end{array}$ \\
\hline $\begin{array}{l}2 \\
8\end{array}$ & $\begin{array}{l}\text { When testing } \\
\text { high-voltage } \\
\text { cables, high } \\
\text { voltage cables } \\
\text { were not } \\
\text { discharge }\end{array}$ & $\begin{array}{l}6 \\
0 \\
1 . \\
3 \\
2\end{array}$ & $\begin{array}{c}19 \\
63 \\
.3 \\
2\end{array}$ & $\begin{array}{l}6 \\
5 \\
2 . \\
6 \\
8\end{array}$ & $\begin{array}{l}3 \\
2 \\
7 . \\
7 \\
5\end{array}$ & $\begin{array}{c}47 \\
5 . \\
28\end{array}$ & 7.37 \\
\hline $\begin{array}{l}2 \\
9\end{array}$ & $\begin{array}{c}\text { When } \\
\text { overhaul } \\
\text { high-voltage } \\
\text { electrical, not } \\
\text { mount ground } \\
\text { or cut power, } \\
\text { just push out } \\
\text { handcart }\end{array}$ & $\begin{array}{l}5 \\
5 \\
0 . \\
2 \\
2\end{array}$ & $\begin{array}{c}18 \\
08 \\
.1 \\
8\end{array}$ & $\begin{array}{l}7 \\
7 \\
9 . \\
9 \\
0\end{array}$ & $\begin{array}{l}4 \\
1 \\
5 . \\
4 \\
2\end{array}$ & $\begin{array}{c}32 \\
3 . \\
37\end{array}$ & 5.58 \\
\hline $\begin{array}{l}3 \\
0\end{array}$ & $\begin{array}{l}\text { While shuttle } \\
\text { car and } \\
\text { forklift car are } \\
\text { walking, } \\
\text { hands or arms } \\
\text { of drivers } \\
\text { exposed } \\
\text { outside the } \\
\text { cab }\end{array}$ & $\begin{array}{l}4 \\
6 \\
1 . \\
2 \\
3\end{array}$ & $\begin{array}{l}18 \\
55 \\
.1 \\
5\end{array}$ & $\begin{array}{l}7 \\
8 \\
3 . \\
0 \\
0\end{array}$ & $\begin{array}{l}3 \\
3 \\
4 . \\
4 \\
5\end{array}$ & $\begin{array}{l}18 \\
7 . \\
64\end{array}$ & 2.39 \\
\hline
\end{tabular}

Under the circumstances of considering positive and negative, $f$ value was larger, which represents a higher relative risk of the near miss event, the more attention should be paid. For example the 18th events, where, employee assignments was unreasonable, and coordinating work was not in place, so the $f$ value was -27.28 , it was the relatively less risky events. After getting $f$ values of 30 samples, a curve was drawn, and it was shown in Figure 3.

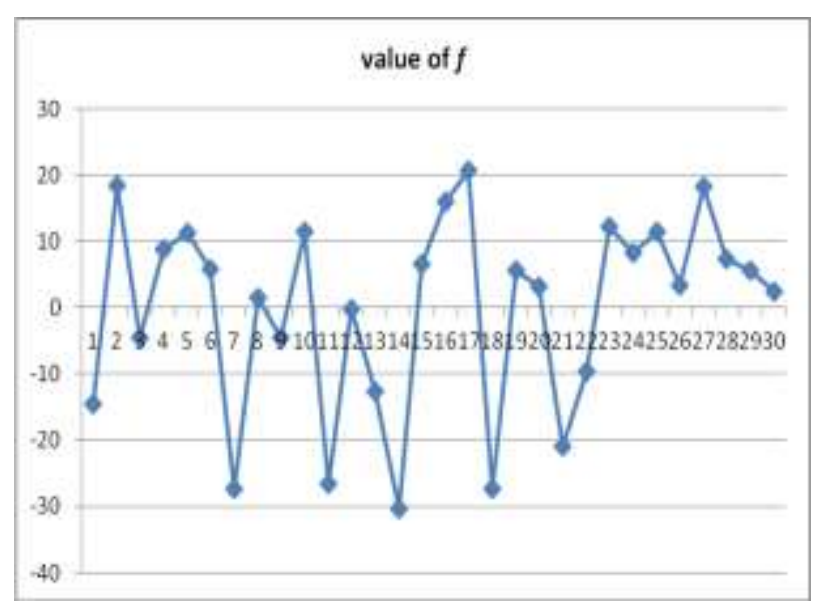

Figure 3. The $f$ values of 30 near miss

Based on $f$ index distribution in Figure 2.1, the relative risk degree of near miss can be divided into seven intervals: $f<-30,(-30,-20],(-20,-10],(-10,0],(0,10],(10,20]$ and $f>20$. Their corresponding risk levels are basic non-risk, small risk, less risk, general risk, relatively large risk, large risk and great risk.

Just as can be seen in Figure 3, No. 2, 5, 10, 16, 23, 25 and 27 near miss falls within $(10,20]$ interval, which indicated that seven near miss was worthy of attention, it belong to large risk and should be promptly stopped. Especially for No. 17 near miss, its $f$ value is greater than 20 , it belong to large risk event, and it should be strictly prohibited in the mine.

To further verify the accuracy of the results, this method was compared with the traditional LEC risk assessment methods, and the result was shown in Figure 4, we found that their result is basically the same, these objective data with describe of mine workers further proved the obtained experimental results.

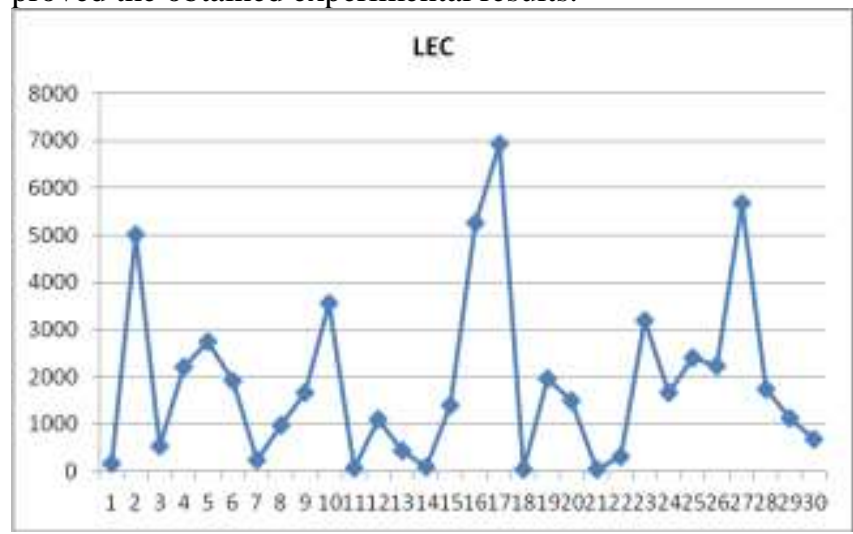

Figure 4. . LEC score of near miss 
Such refined classification make employees have a more profound and accurate understanding on near miss events. By classification, the resources can be sufficiently and rationally utilized, simultaneously it can avoid waste, which is the effective pre-control method to avoid a major accident. In the coal industry, Wei Xiangrong raised that people's unsafe behavior is the biggest risk of accidents in 2011. Unexpected accidents often occurred on the basis of many times violations, the occurrence of an accident have repeatedly attempted events. The best way to prevent accidents is to eliminate the "three violations" and destroy "attempted." In 2007, He Peng proposed any a happened accident has signs to follow for the enterprise, it was the results of a variety of security risks and the risk factors during the producing process. How to detect the risk factors and take measures to eliminate the risk management is the top priority for coal enterprises, and this is the advantages of risk management theory. Therefore, the risk management ideas should be vigorously popularized in the coal enterprise, which help to discover risk factors and take measures in advance. In 2004, Su Liping proposed the cause of accident was people's unsafe behavior and unsafe condition of matter, and unsafe condition of matter was also caused by people's unsafe behavior in the final. Error caused by human was ubiquitous, but not every error caused by human induced failure of the system. Due to the time difference in which the system located, there are a considerable number of errors caused by human which only lead to attempted events or a potential failure, and it often failed to arouse people's full attention, but these potential failure phenomenon may induce potential problems for the safe production. Only by subdividing near miss events into different levels, different attention was pay to different levels, can we prevent possible trouble.

\section{CONCLUSION}

(1) By defining concept of near miss, and analyzing its difference and relationship with hazards and risks, the near miss of coal mine referred to small events that has a characteristic symptom of accidents, but did not produce losses and injuries or only a slight loss and damage due to lack of conditions. If conditions change, there will still be the possibility resulting in an accident.

(2) Learn from LEC risk evaluation, the near miss risk was assessed by using five indicators associated with risk ratio. These five indicators are: the possibility of near miss leading to an accident occurring, L; frequent of personnel exposure to hazardous environments, $\mathrm{E}$; the monitor extent of the event, M; the learn value of events, $\mathrm{S}$; the consequences of an accident occurring, $\mathrm{C}$. The relative risk assessment model of near miss was constructed by using $f$ risk index, practice has proved that the classification results are basically the same with the traditional LEC risk assessment methods, and it can reflect the risk characteristics of near miss events from the many perspectives.

\section{ACKNOWLEDGMENT}

The authors gratefully acknowledge the assistance from the National Natural Science Foundation (71271169, 71273208), Doctoral Fund (20126121110004, 20116121110002) and substantial grants from the special fund of Shaanxi Education Department (12JK0036) for this study. The authors are also grateful to the experts who participated in the expert opinion study for their time and efforts.

\section{REFERENCES}

[1] Cui Zhaohua, "Statistics on the Data of Coal Mine Gas Accidents in our Country between Year 2001-2008 and Causal Analysis," Sci-tech information development \& economy, vol.9, no.21, 2009 , pp.139-141.

[2] Tian Shuicheng, "Research on identification and control of three types of hazards," Doctoral Dissertation, Beijing, Beijing Institute of Technology, 2001.

[3] Shi Xiaohong, "Research on work safety Near miss," Doctoral Dissertation, Beijing, Capital University of Economics and Business, 2010.

[4] Wright, "Advanced Aviation Safety and Aircraft Accident and Incident Investigation," Course, Joint Air Traffic Services, Aug. 2005, pp.23-26,doi:org/10.1016/j.jcfm.2005.08.004.

[5] James R. Phimister, Ulku Oktem, et al. "Near-Miss incident management in the chemical process industry," Risk Analysis, vol.23, Mar.2003, pp. 445-460, doi: 10.1111/1539-6924.00326.

[6] Santos Reyes, Alan N. Beard, "Assessing safety management systems," Journal of Loss Prevention in Process Industries, vol.15,2002, pp.77-95, doi:org/10.1016/S0950-4230(01)00066-3.

[7] Luo Yun, Fan Yunxiao, et al. "Risk Analysis and Safety Evaluation,” Beijing, Chemical Industry Press, Mar.2004.

[8] Fan Jianhui, "Research on great accident hazard and classification method underground mines," Master Dissertation, Kunming University of Science and Technology, 2006.

[9] U Ritwik, "Risk-based approach to near miss," Hydrocarbon processing, Oct.2002,pp.93-96, doi.org/10.1016/j.jcfm.2005.08.004.

[10] David Best, Siobhan Havis,J. J. Payne-James, et al. "Near miss incident in police custody suites in London in 2003: a feasibility study," Journal of Clinical Forensic Medicine, vol.13,2006,pp.6064. 